\title{
HIGHLIGHTS
}

PROSTATE CANCER

\section{Cadherin 2: an important new player in castration resistance}

The predominant cause of death in men with prostate cancer is metastatic disease that has become resistant to androgen deprivation therapy (ADT). The transition to castration resistance seems to be mediated partly by reactivation of androgen receptor signaling, but other mechanisms that act independently of the androgen receptor might also have important roles. In a paper published in Nature Medicine, Tanaka and colleagues report the results of a series of experiments that identified cadherin 2 (CDH2; also known as $\mathrm{N}$-cadherin) as having an important role in prostate cancer progression and castration resistance, and also as a potentially valuable therapeutic target in this malignancy.

Gene expression analysis of androgendependent and castration-resistant prostate cancer xenografts revealed markedly higher expression of $\mathrm{CDH} 2$ in the castration-resistant grafts. Next, tissue samples from patients with benign prostatic hyperplasia (BPH), ADT-naive prostate cancer, prostate cancer treated for 3-9 months with neoadjuvant ADT, or castration-resistant prostate cancer were immunohistochemically stained for $\mathrm{CDH} 2$ expression. $\mathrm{CDH} 2$ expression was rare in $\mathrm{BPH}$ ( $1 \%$ of cells stained) but increased with the duration of androgen deprivation, being highest in castration-resistant prostate cancer ( $41 \%$ of cells stained).

Androgen-dependent prostate cancer cell lines ectopically expressing $\mathrm{CDH} 2$ showed increased invasiveness and metastatic potential when implanted subcutaneously into mice. Furthermore, these cell lines were also capable of proliferating in the absence of androgen in vitro and forming tumors in vivo in castrated mice.

To test whether $\mathrm{CDH} 2$ might be a useful therapeutic target in advanced prostate cancer, castration-resistant cell lines were treated in vitro with anti-CDH2 antibodies. The antibodies inhibited invasion, attachment and proliferation of prostate cells. Treatment of castrated mice carrying palpable castration-resistant tumors with the anti-CDH2 antibodies markedly decreased tumor growth, invasion of underlying muscle and the occurrence of lymph node metastasis. In addition, anti-CDH2 antibody treatment delayed the emergence of castration resistance in androgen-dependent tumors.

The authors conclude that their results indicate important roles of $\mathrm{CDH} 2$ in prostate cancer invasion, metastasis and castration resistance. These processes can be suppressed or delayed by specific anti-CDH2 antibodies. $\mathrm{CDH} 2$, therefore, represents a promising new therapeutic target in prostate cancer, although, as $\mathrm{CDH} 2$ is widely expressed in normal tissues, the toxicity of anti-CDH2 antibodies will require careful study before it can be safely tested in clinical trials.

Nick Warde
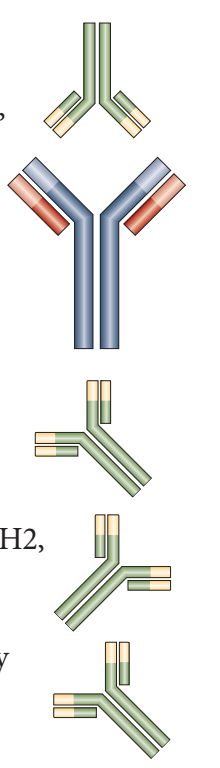

article Tanaka, H. et al. Monoclonal antibody targeting of $\mathrm{N}$-cadherin inhibits prostate cancer growth, metastasis and castration resistance. Nat. Med. doi:10.1038/nm.2236 